\title{
Lift Generation of an Elliptical Airfoil at a Reynolds Number of 1000
}

\author{
S. Tobing \\ Department of Mechanical Engineering, Faculty of Engineering, \\ Atma Jaya Catholic University of Indonesia, Jalan Jenderal Sudirman 51, \\ Jakarta, Indonesia 12930 \\ Email: sheila.tobing@atmajaya.ac.id
}

\begin{abstract}
Bumblebees cannot fly! That conclusion is likely to be drawn by scientists who analysed the insect using aerodynamics of stationary wings such as that of a passenger aircraft. Looking at the insect again using a newfound understanding of unsteady aerodynamics; it is clear why bumblebees can fly. Bumblebees utilise mechanisms behind unsteady aerodynamics such as leading-edge vortices (LEVs) formation, wake capture, and rapid end-of-stroke rotation to generate forces that enable the insect to fly. This study focuses on two-dimensional (2D) elliptical airfoil. Earlier works found the aerodynamic characteristics of an elliptical airfoil to differ greatly from a conventional airfoil, and that this airfoil shape could generate the counter-rotating vortices used by insects to generate lift. Therefore, this research aims to study the lift generation of a bumblebee-inspired elliptical airfoil in a normal hovering flight. This study focuses on hovering flight with the insect flies in a nearly stationary position, which explains the importance of lift generation to stay aloft. The motion of the elliptical airfoil is inspired by the flapping kinematics of bumblebees at a typical Reynolds number range of $\mathrm{Re}=\mathrm{O}\left(10^{3}\right)$. It is found that the current two-dimensional model is capable of capturing the counter-rotating vortices and correlates the formation of these structures to a high production of lift. These results show that bumblebees utilise these counter-rotating vortices to generate lift enough to fly in hovering flight. This results also indicate that flapping 2D elliptical airfoils can be used to investigate their 3D wing counterparts, which translate to a reduced time and computing costs.
\end{abstract}

Keywords: Flapping wing; elliptical airfoil, bumblebee wings, counter-rotating vortex; lift generation.

\section{INTRODUCTION}

Interests in insect flight have increased steadily in the last three decades. This increase is sparked by the natural interests of human to flying and the potential applications of insect flight to the design of macro air vehicles and more recently tidal turbines. Previous researchers discovered the key phenomena of insect flight, for example, leading-edge vortex, added mass and wing-wake interactions [1]. Leading edge vortex occurs when the flow on the wing separates as it passes the leading edge and reattaches before the trailing edge [2]. The leading edge vortex results in the increase in lift and delayed stall. Added mass is a phenomenon where the wing during acceleration experiences a reaction force because of the accelerated fluid [1,3]. Wing-wake interaction is when a wing interacts with vortices shed in the previous strokes, which causes the rapid change in forces right after supination and pronation [4]. 
Flapping wings with various shapes, for example, rectangular [5,6], elliptical $[7,8]$, and Zimmerman $[9,10]$ had been studied frequently. Simulations of these threedimensional (3D) wings are ideal for capturing the flow phenomena around insect/flapping wings. However, earlier works have shown that two-dimensional (2D) calculations provide useful information about the flow and can serve as a reliable and practical tool to predict the forces generated by a flapping three-dimensional wing $[11,12]$. Many types of 2D objects were studied in the past. Several of the most frequently analysed were NACA airfoils [13-15] and flat plates [16-18].

Bumblebees are pollinators that fly in the Reynolds number regime of $\mathcal{O}\left(10^{3}\right)$. Bumblebees are known to have a high wing loading of $0.17 \frac{\mathrm{g}}{\mathrm{cm}^{2}}$, which translates to their ability to fly while carrying heavy load despite of having small wings [19]. This capability to fly while lifting heavy load is beneficial for MAVs especially those developed to deliver goods. One vortex ring around each wing was observed by Bomphrey, et al. [20] during experiments using smoke-line visualization techniques on free-flying bumblebees at $1.2 \mathrm{~m} / \mathrm{s}(\operatorname{Re}=2500)$. Bomphrey, et al. [20] observed that this vortex structure was formed because bumblebees shed not only their tip vortices but also their root vortices. The ring vortices on 3D bumblebee wings correspond to the counter-rotating vortices on 2D airfoils.

Earlier studies found counter-rotating vortices around an elliptical airfoil. Wang [21] numerically study an elliptical airfoil (2D) hovering under a figure-eight motion and found that counter-rotating vortices were a product of LEVs and trailing-edge vortices (TEVs), and not tip vortices which is a phenomenon found on three-dimensional wings. Poelma et al. [22] studied a dynamically-scaled wing moving in mineral oil and observed a pair of counter-rotating structures $\left(\operatorname{Re}=\frac{V_{t i p} c}{v}\right)$. Wei, et al. [23] numerically studied elliptical airfoils at Reynolds numbers ranging from $10^{4}$ to $10^{6}$ and they also captured vortex dipoles in the flow visualization. The current work extends these published works by implementing the kinematics of bumblebees on the elliptical airfoil.

This work aims at testing 2D elliptical airfoil in the typical Re regime of 1000 . A successful finding of counter-rotating vortices on the elliptical airfoil suggests the adequacy of $2 \mathrm{D}$ model to give insight on the vortex rings on bumblebee wings (3D). Future works on bumblebee wings can be done using a 2D model, which can reduce the time and computing resources.

\section{VALIDATION}

The current solver and setup are validated against the computational data of [24]. The validation case is of an elliptical airfoil that flaps in a normal-hovering mode $\left(\beta=0^{\circ}\right)$ at $\operatorname{Re}$ of 157 (Figure 1). The airfoil flaps with flapping kinematics comprise translational and rotational motion that is described by Eq. (1) and Eq. (2) respectively.

$$
\begin{aligned}
& {[x(t), y(t)]=\frac{A_{0}}{2}(1+\cos 2 \pi f t)(\cos \beta, \sin \beta)} \\
& \alpha(t)=\alpha_{0}+B \sin (2 \pi f t+\varphi)
\end{aligned}
$$


The geometry of the model and the flow conditions are listed in Table 1 . The nondimensional parameters are the ratio of flapping/translation amplitude to chord $=\left(\mathrm{A}_{0} / \mathrm{c}\right)=2.5$ and the ratio of thickness to chord $=(\mathrm{t} / \mathrm{c})=0.125$.

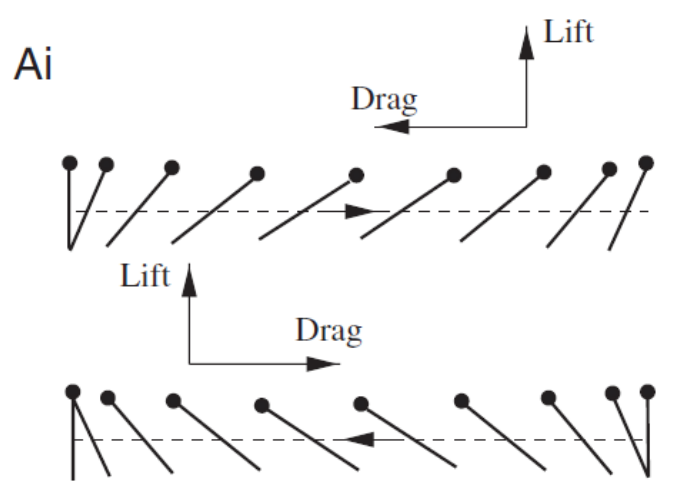

Figure 1. Normal hovering where the stroke plane is flat $\left(\beta=0^{\circ}\right)$ (Figure 2Ai in [24]). The leading edge of the airfoil is shown by the black circle. The top row is upstroke, and the bottom row is downstroke. The direction of lift is perpendicular to the stroke plane, which is the longitudinal plane, and the drag is parallel to the stroke plane.

Table 1. Geometry and flow conditions of the validation case.

\begin{tabular}{ll}
\hline Variable & Value \\
\hline $\mathrm{c}$ & $0.01670 \mathrm{~m}$ \\
$\mathrm{~A}_{0}$ & $0.04175 \mathrm{~m}$ \\
$f$ & $1 \mathrm{~Hz}$ \\
$B$ & $45^{\circ}$ \\
$\phi$ & $0^{\circ}$ \\
$\beta$ & $0^{\circ}$ \\
$\alpha_{0}$ & $90^{\circ}$ \\
$v$ & $1.4 \mathrm{e}-05\left(\mathrm{~m}^{2} \mathrm{~s}^{-1}\right)($ air $)$ \\
$\operatorname{Re}$ & 157 \\
\hline
\end{tabular}

The first cell height of the boundary layer is $1 \times 10^{-5} \mathrm{~m}$ with a growth rate (= the ratio between the height of layer $(n+1)$ to layer $n)$ of 1.1 . The number of layer in the boundary region is 26 , and the total boundary thickness is around $10 \%$ of the chord. The inner region has a growth rate of 1.1 and a maximum size of $0.002 \mathrm{~m}$ with several cells of 28,436 . The outer region has a growth rate of 1.1 with a maximum size of $0.004 \mathrm{~m}$ with several cells of 34,694 .

The validation results are shown in Figure 3. Reasonable agreement is found between the current analysis and the reference data [24]. The first set of testing of the normal hovering airfoil $\left(\alpha_{o}=90^{\circ}, \beta=0^{\circ}\right)$ shows instability. For normal hovering, the airfoil's angle-of-attack $(\alpha)$ varies between $45^{\circ}$ and $90^{\circ}$, which make the airfoil a bluff body. Unsteadiness was observed on flow over bluff body at Re of 150 [25], and this might be the reason behind the difficulty in predicting the forces and thus the slight difference between the current simulation and the results of Wang [24]. The same validation case is used in another work on the drag production of an elliptical airfoil [26]. 


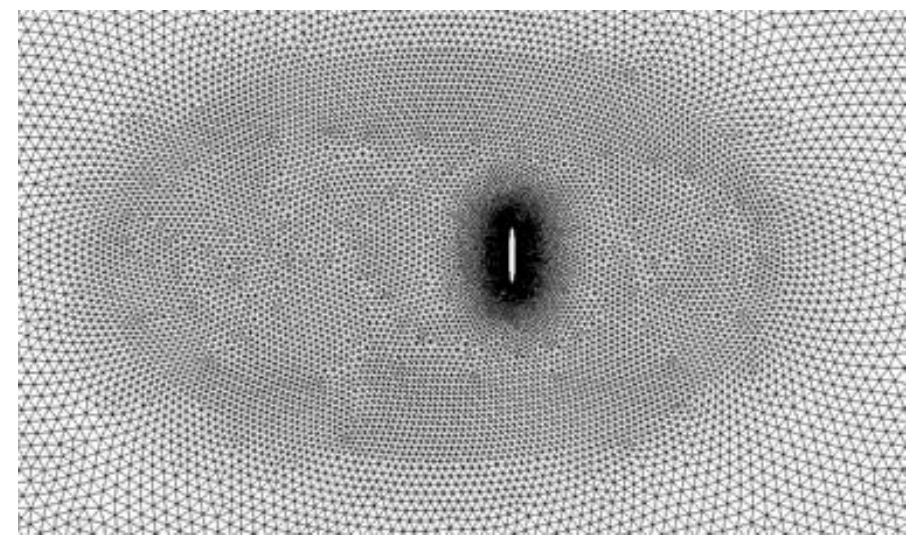

(a)
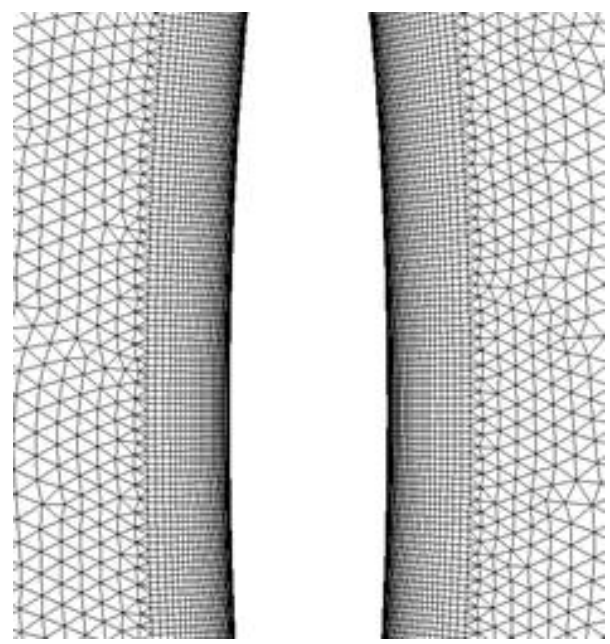

(b)

Figure 2. Computational model: (a) Grid of the whole domain, and (b) Zoom on the boundary layer and grid around the elliptical airfoil.

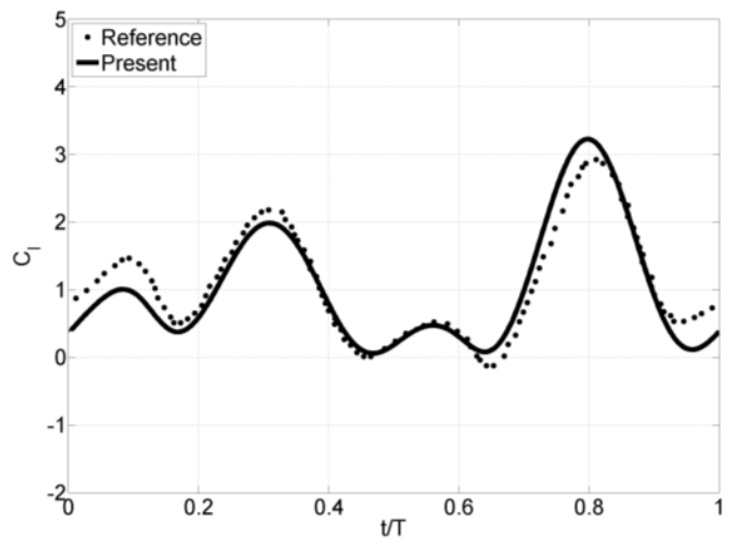

(a)

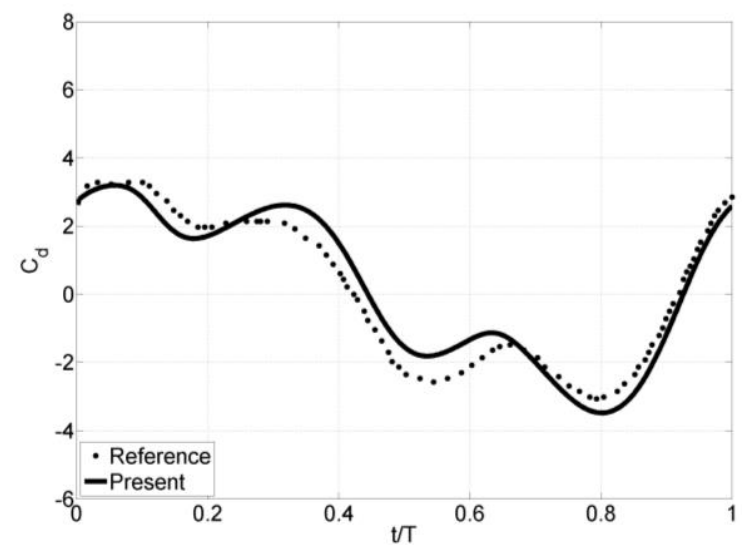

(b)

Figure 3. (a) Lift and (b) drag coefficients of the current model in comparison to the reference [24]. 


\section{NUMERICAL MODELING OF ELLIPTICAL AIRFOIL}

\section{Flow Conditions}

Bumblebee's flight at $\mathrm{Re}=1360$ with Re defined as $\frac{\phi \mathrm{fS}}{v}$ has the following parameters (Table 2) to describe its flapping motion [27].

Table 2. Kinematics of a real bumblebee.

\begin{tabular}{ll}
\hline Variable & Value \\
\hline$\phi$ & $112^{\circ}$ \\
$f$ & $145 \mathrm{~Hz}$ \\
$S$ & $5.2 \times 10^{-5} \mathrm{~m}^{2}$ \\
$v$ & $1.46 \mathrm{e}-05 \mathrm{~m}^{2} \mathrm{~s}^{-1}$ (air) \\
\hline
\end{tabular}

The calculation of Eq. (3) results in the following flow conditions:

$$
\frac{\phi \mathrm{fS}}{v(\text { air })}=\frac{\pi \mathrm{fA}_{0} \mathrm{c}}{v}
$$

While keeping the frequency, flapping amplitude and chord the same as the validation case, the kinematic viscosity is adjusted to reach a Reynolds number of 1000 where bumblebees commonly fly in Table 3 . The ratio of translation amplitude to chord $\left(=\frac{A_{0}}{c}\right)$ is 2.5 and the ratio of thickness to chord $\left(=\frac{t}{c}\right)$ is 0.125 .

The flapping motion of the elliptical airfoil is described by two equations, one each for pitching $(\dot{\alpha})$ and flapping angle rate $(\dot{\phi})$ as in Eq. (4) and Eq. (5). The elliptical airfoil maintains a constant angle-of-attack during the downstroke $\left(\alpha_{D}\right)$ and upstroke $\left(\alpha_{U}\right)$ (Table 3). The duration of wing rotation $\left(\Delta \tau_{\mathrm{r}}\right)$ is $0.22 \mathrm{~T}$ with $T$ is the period of one flapping cycle/wingbeat $\left(\mathrm{T}=\frac{1}{\mathrm{f}}\right)$. The non-dimensional time is $\tau=\frac{\mathrm{t}}{\mathrm{T}}$ and $\tau_{\mathrm{D}}$ is the start of wing rotation during the downstroke. For the upstroke, $\tau_{\mathrm{D}}$ is substituted with $\tau_{\mathrm{U}}$ and the sign of $\dot{\alpha}_{0}$ is reversed, see Eq. (6).

$$
\begin{aligned}
& \dot{\phi}=\pi \mathrm{fA}_{0} \cos (2 \pi \mathrm{ft}) \\
& \dot{\alpha}=0.5 \dot{\alpha}_{0}\left\{1-\cos \left(\frac{2 \pi\left[\tau-\tau_{\mathrm{D}}\right]}{\Delta \tau_{\mathrm{r}}}\right)\right\} \\
& \dot{\alpha}_{0}=\left(\frac{\pi-\alpha_{\mathrm{U}}-\alpha_{\mathrm{D}}}{\Delta \tau_{\mathrm{r}} \times \mathrm{T}}\right)
\end{aligned}
$$

More details on the bumblebee flapping kinematics can be found in previous study [28].

\section{Computational Model}

The first cell height of the boundary layer is $1 \times 10^{-5} \mathrm{~m}$ with a growth rate of 1.1 . The number of layer in the boundary region is 20 , and the total boundary thickness is around 
$5 \%$ of chord. The inner region has a growth rate of 1.1 and a maximum size of $0.0015 \mathrm{~m}$ with several cells of 26,124 . The outer region has a growth rate of 1.1 with a maximum size of $0.01 \mathrm{~m}$ with several cells of 9,318 .

Table 3. Kinematics of bumblebee-inspired flapping elliptical airfoil.

\begin{tabular}{ll}
\hline Variable & Value \\
\hline$c$ & $0.01670 \mathrm{~m}$ \\
$A_{0}$ & $0.04175 \mathrm{~m}$ \\
$f$ & $1 \mathrm{~Hz}$ \\
$\phi$ & $0^{\circ}$ \\
$\beta$ & $0^{\circ}$ \\
$\alpha_{D}$ & $58.8^{\circ}$ \\
$\alpha_{U}$ & $49.3^{\circ}$ \\
$v$ & $2.19 \mathrm{e}-06 \mathrm{~m}^{2} \mathrm{~s}^{-1}$ \\
$\operatorname{Re}$ & 1000 \\
\hline
\end{tabular}

\section{LIFT GENERATION AT REYNOLDS NUMBER 1000}

It is commonly found in other papers that flapping airfoil/wing models are run for multiple cycles to ensure the unsteady flow is resolved properly [12,29]. The current simulations need to complete 12 cycles before achieving a repetitive time-history of forces (Figure 5). The time-averaged lift coefficient $\left(\bar{C}_{l}\right)$ of the last cycle is 0.6366 .

To show the amount of lift force generated by a bumblebee wing, the individual bumblebee named BB01 whose wing motion is used in the current calculations with a mass of $175 \mathrm{mg}$, a wing length of $1.32 \mathrm{~cm}$ and a wing area of $1.0597 \mathrm{~cm}^{2}$ is chosen as an example [27]. This bumblebee flaps with a wing-beat frequency of $145 \mathrm{~Hz}$ and a stroke amplitude of $112^{\circ}$. This value of $\bar{C}_{l}$ is equal to a lift force production of $3.6342 \times 10^{-3}$ $\mathrm{N}$ for one wing and $1.4536 \times 10^{-2} \mathrm{~N}$ for two wings. This amount of lift is enough to counter the weight of the bumblebee of $1.7167 \times 10^{-3} \mathrm{~N}$ and any additional load such as pollen. This finding shows how bumblebees can fly by utilising unsteady aerodynamic mechanisms most prominently leading edge and trailing edge vortices.

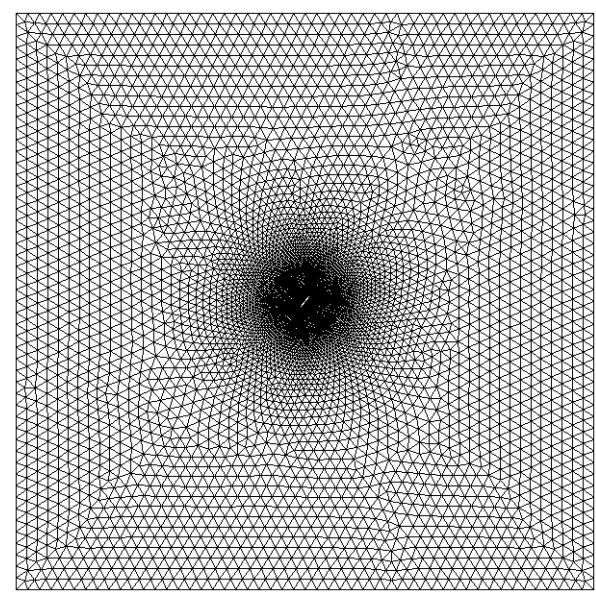

(a) 


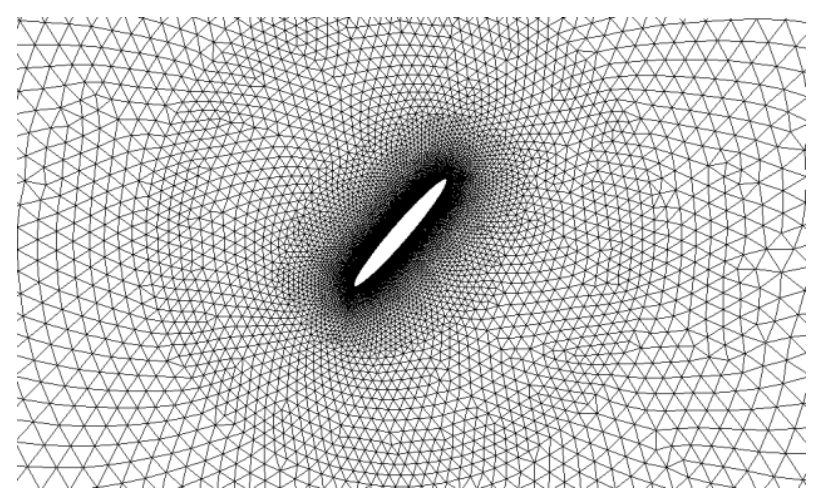

(b)

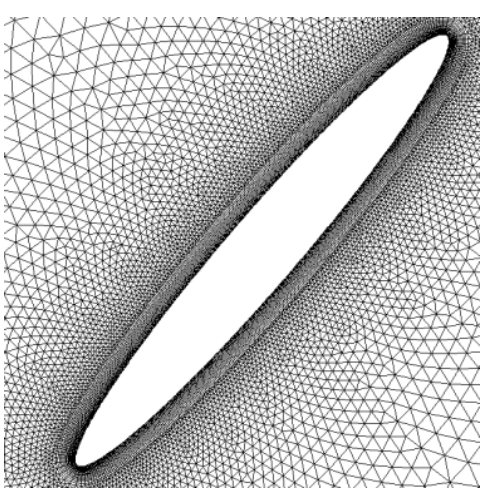

(c)

Figure 4. Computational domain: (a) Computational domain with boundary layer and enclosing circle, (b) zoom in on the circle and; (c) boundary layer and airfoil move together as a rigid body.

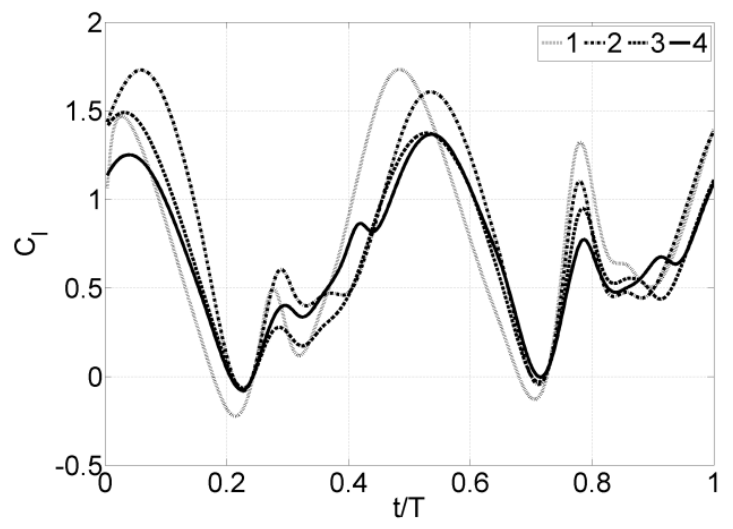

(a)

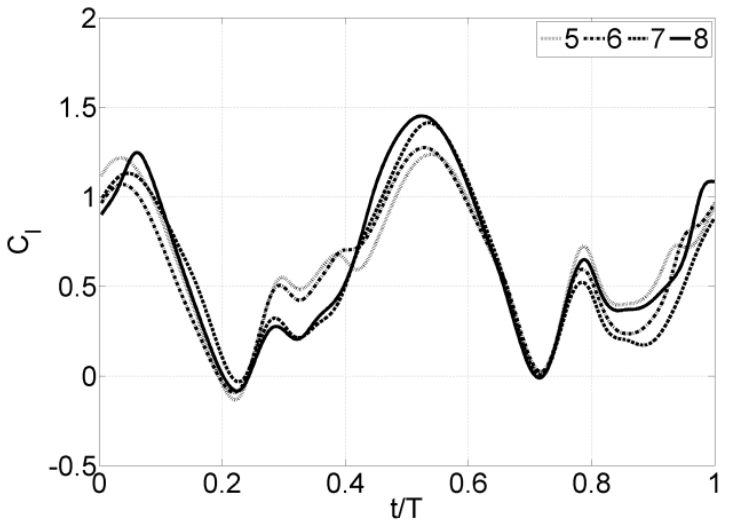

(b)

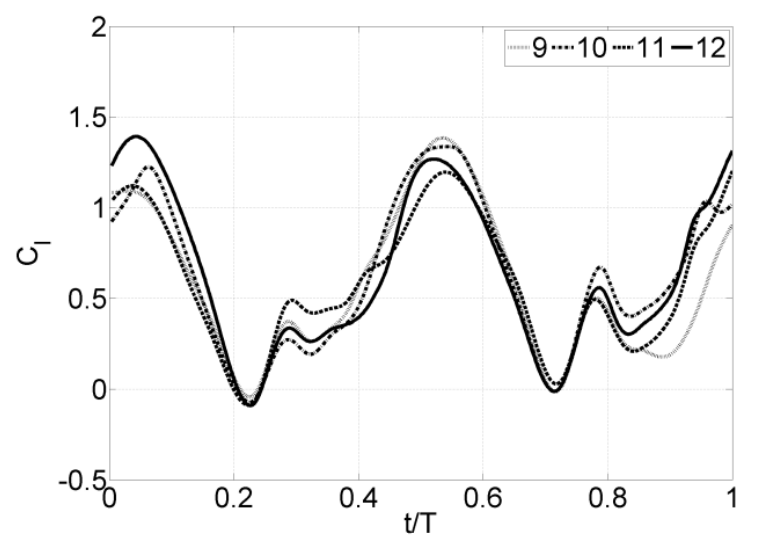

(c)

Figure 5. Time history of lift coefficients for (a) cycle 1 to 4 , (b) cycle 5 to 8 and; (c) cycle 9 to 12 .

Only the time history until mid-cycle (downstroke) is explained due to the similar pattern of lift coefficient in the remaining half of the cycle. The description is divided into two: from 0.04 to 0.2 , where the lift decreases from the global maximum to minimum and from 0.2 to 0.52 , where the lift increases to a local maximum (Figure 6). 


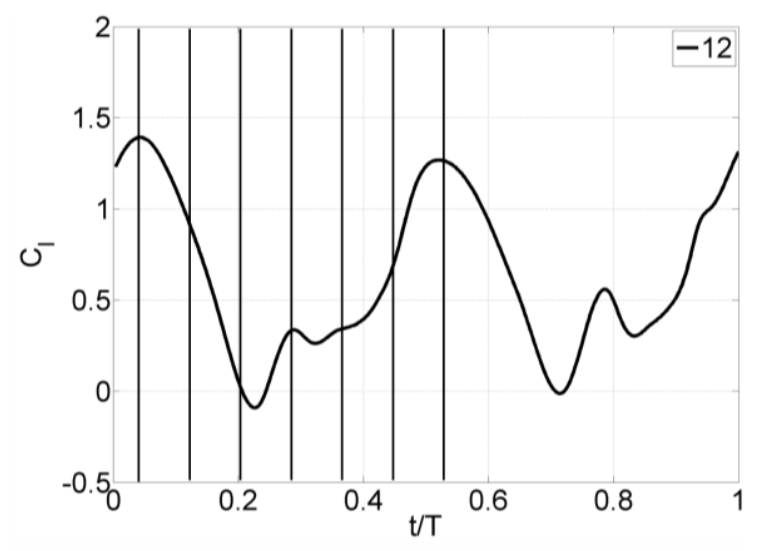

Figure 6. Time history of lift coefficients. From left to right are lines marking $\frac{t}{T}$ of 0.04 to 0.52 with a 0.08 interval.

At 0.04 a cycle, a leading-edge vortex (LEV) is created and still attached to the upper surface of the elliptical airfoil as shown in Figure 7(a). This LEV creates a lowpressure region (blue area) on the upper surface, which in combination with the higher pressure (green-yellow-orange area) on, the lower surface results in the global maximum of lift $\left(\mathrm{C}_{1}=1.3923\right)$. At 0.12 of Figure $7(\mathrm{~b})$, this LEV begins to detach from the surface as the airfoil rotates in a counterclockwise direction. The LEV detaches even more at 0.2 , which is shown by the blue region moves further away from the airfoil's upper surface and is reflected in the near zero lift $\left(\mathrm{C}_{1}=0.0549\right)$ at this time of the cycle.

From 0.28 to 0.36 of in Figure 7(d) and 7(e), the LEV shed into the surrounding flow and at the same time being pushed further by the rotating/pitching airfoil. At 0.36, a trailing-edge vortex begins to develop. At 0.44 in Figure 7(f), counter-rotating vortices appear when a new LEV develops, and the TEV continues to grow. As the LEV and TEV grow, so does the low-pressure region on the upper surface of the airfoil and the lift increases.

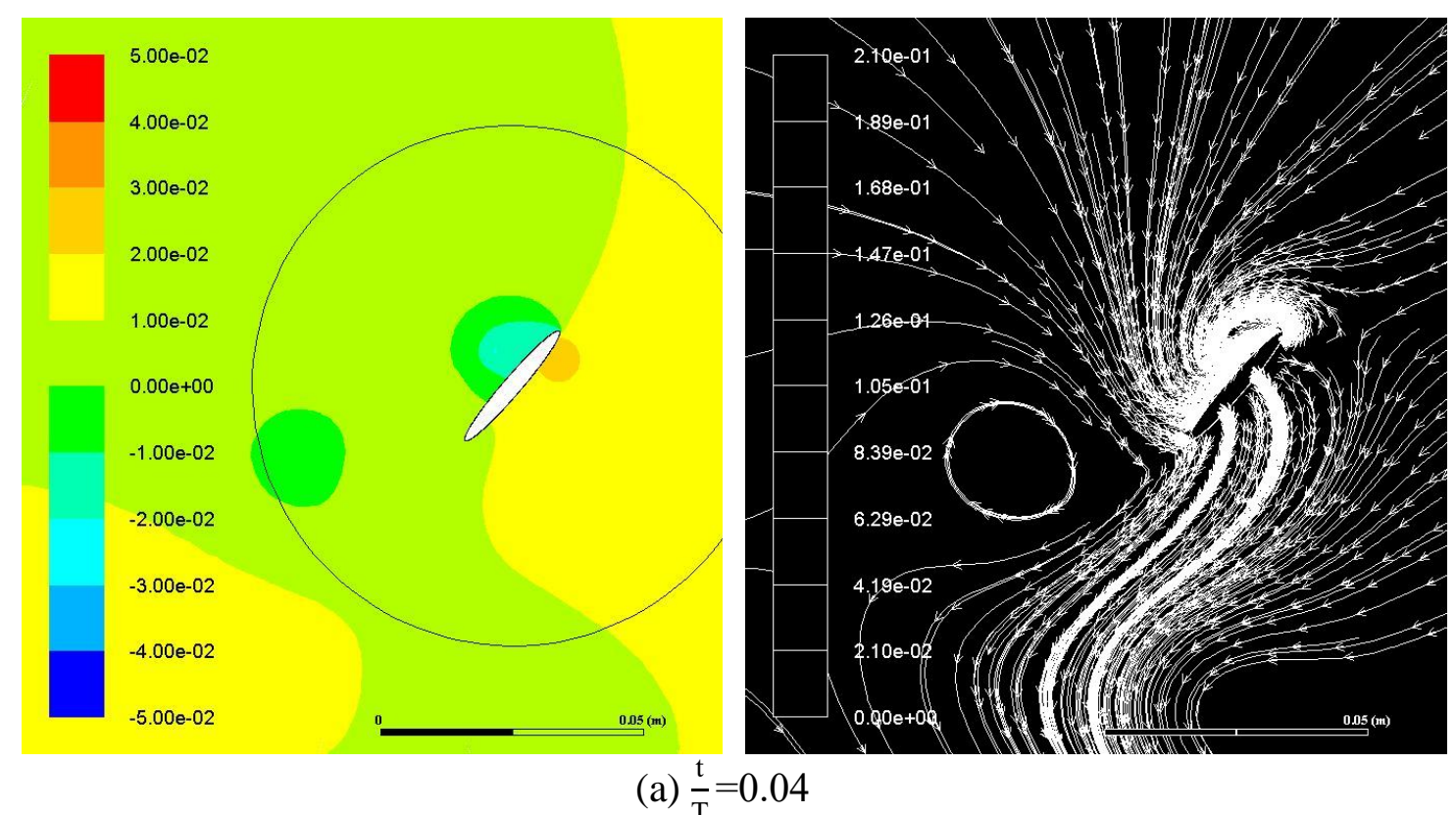



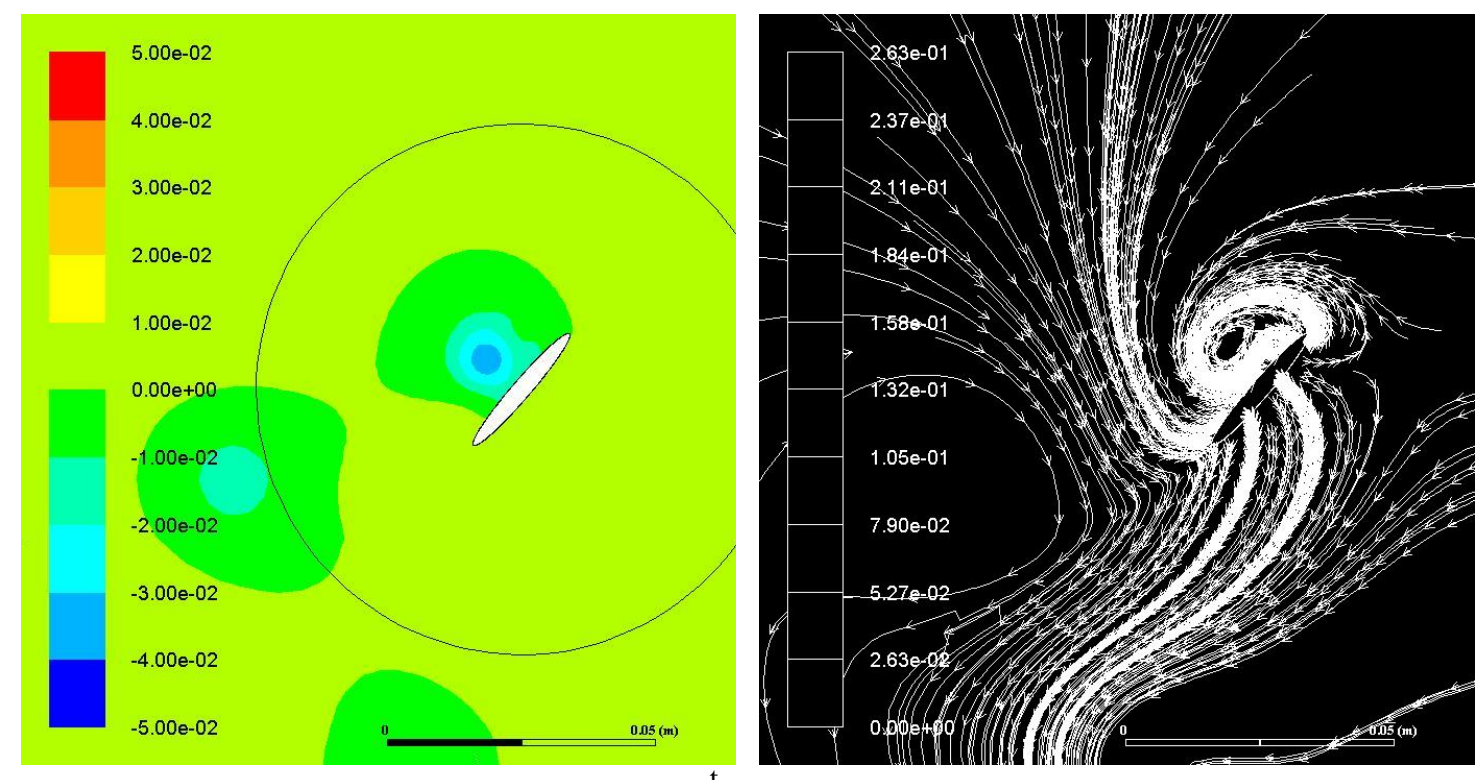

(b) $\frac{\mathrm{t}}{\mathrm{T}}=0.12$

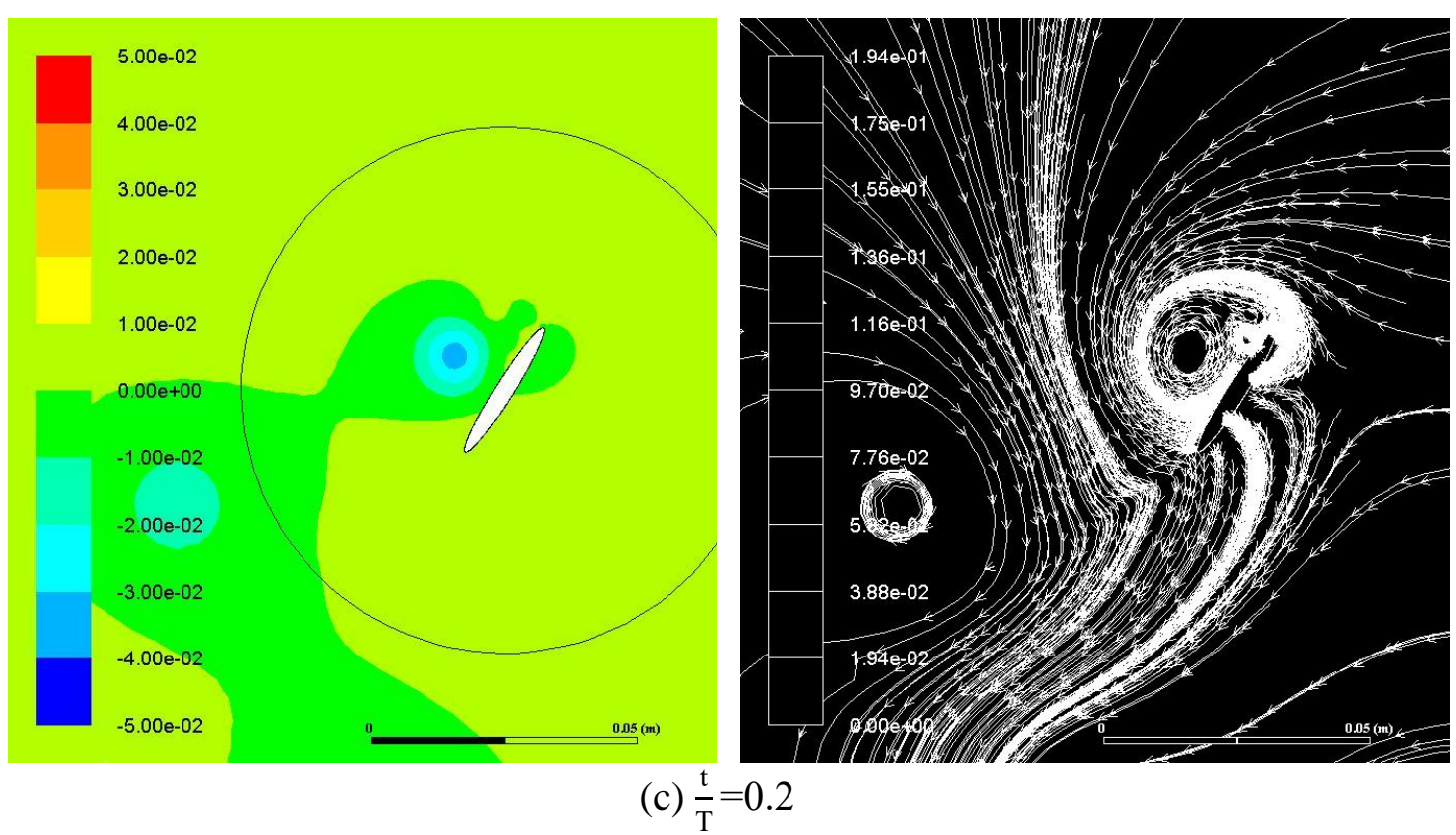



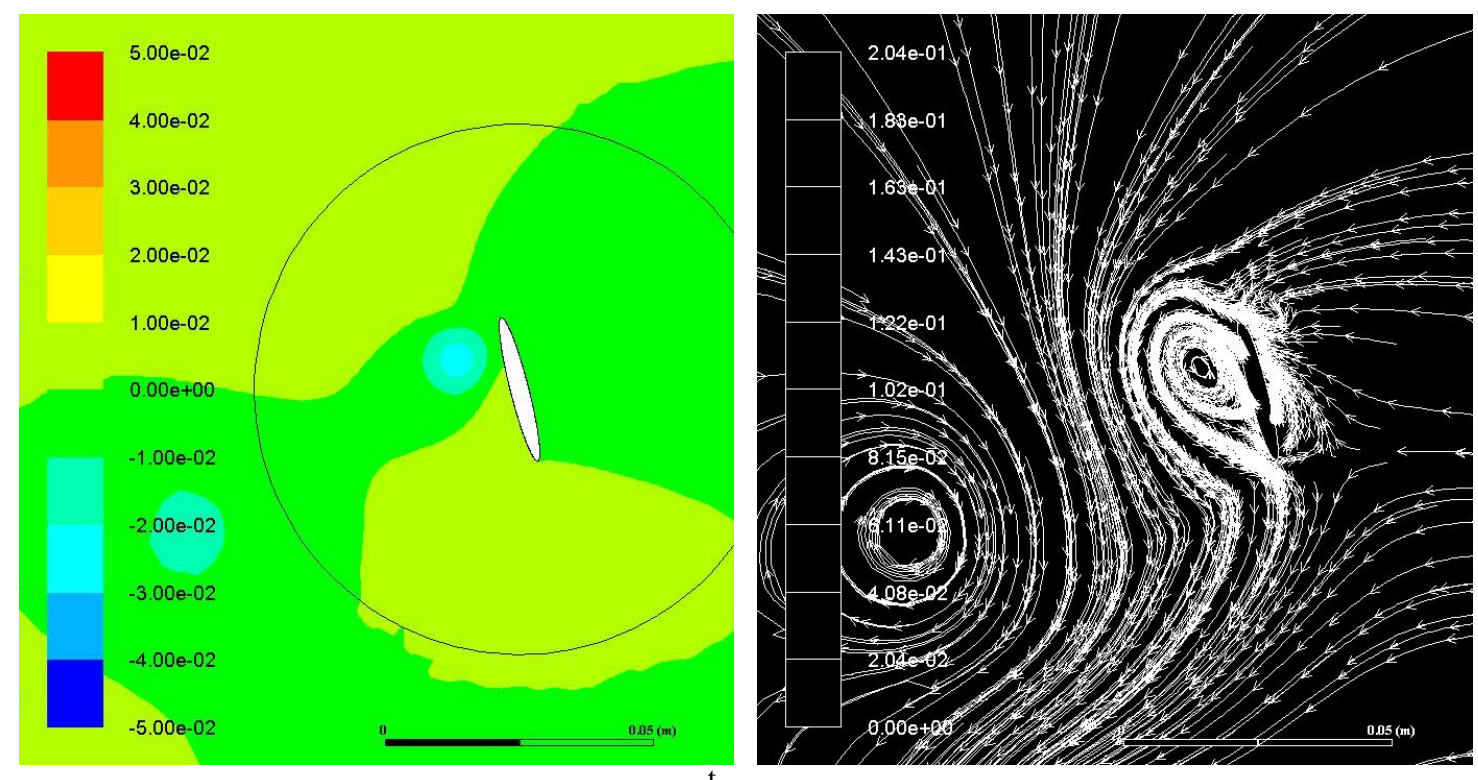

(d) $\frac{\mathrm{t}}{\mathrm{T}}=0.28$
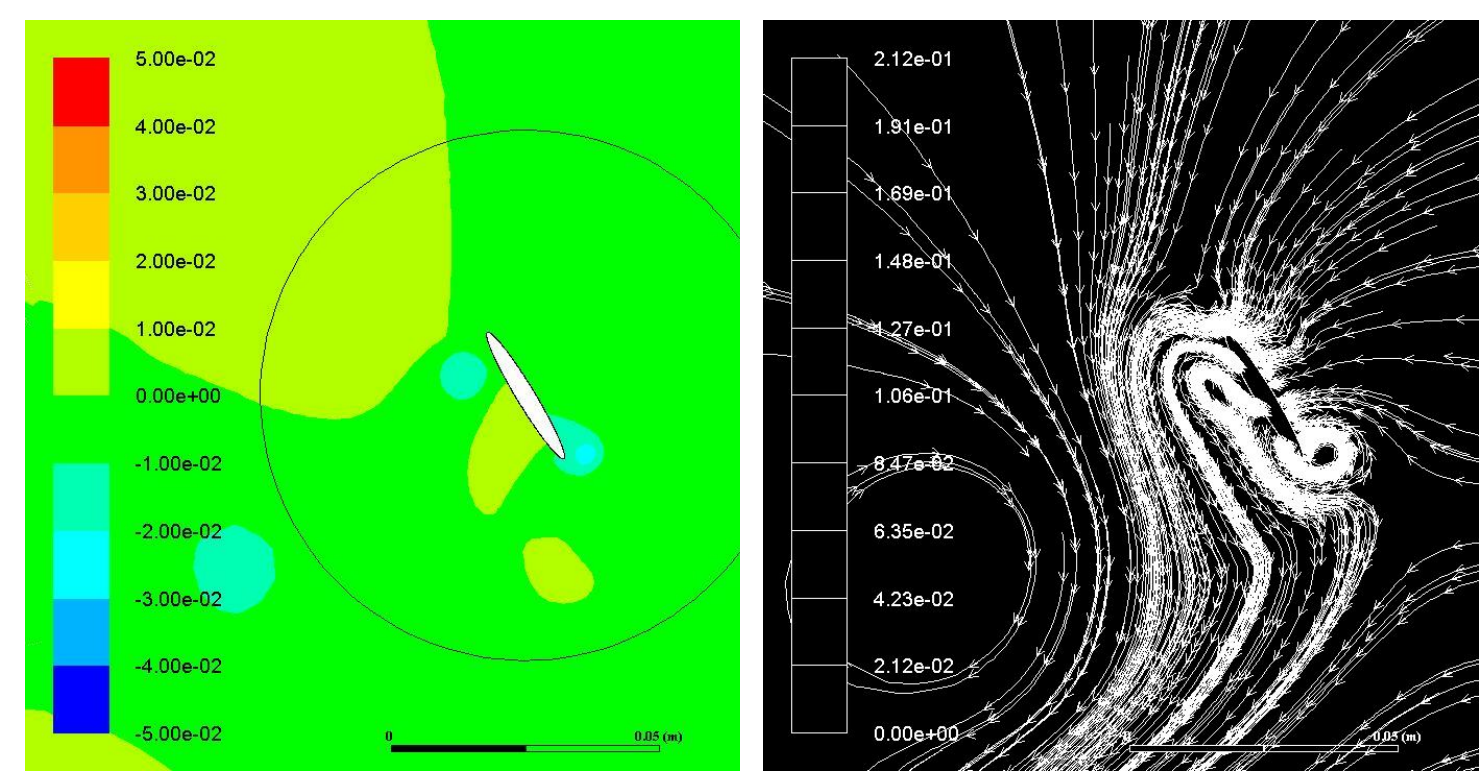

(e) $\frac{\mathrm{t}}{\mathrm{T}}=0.36$ 

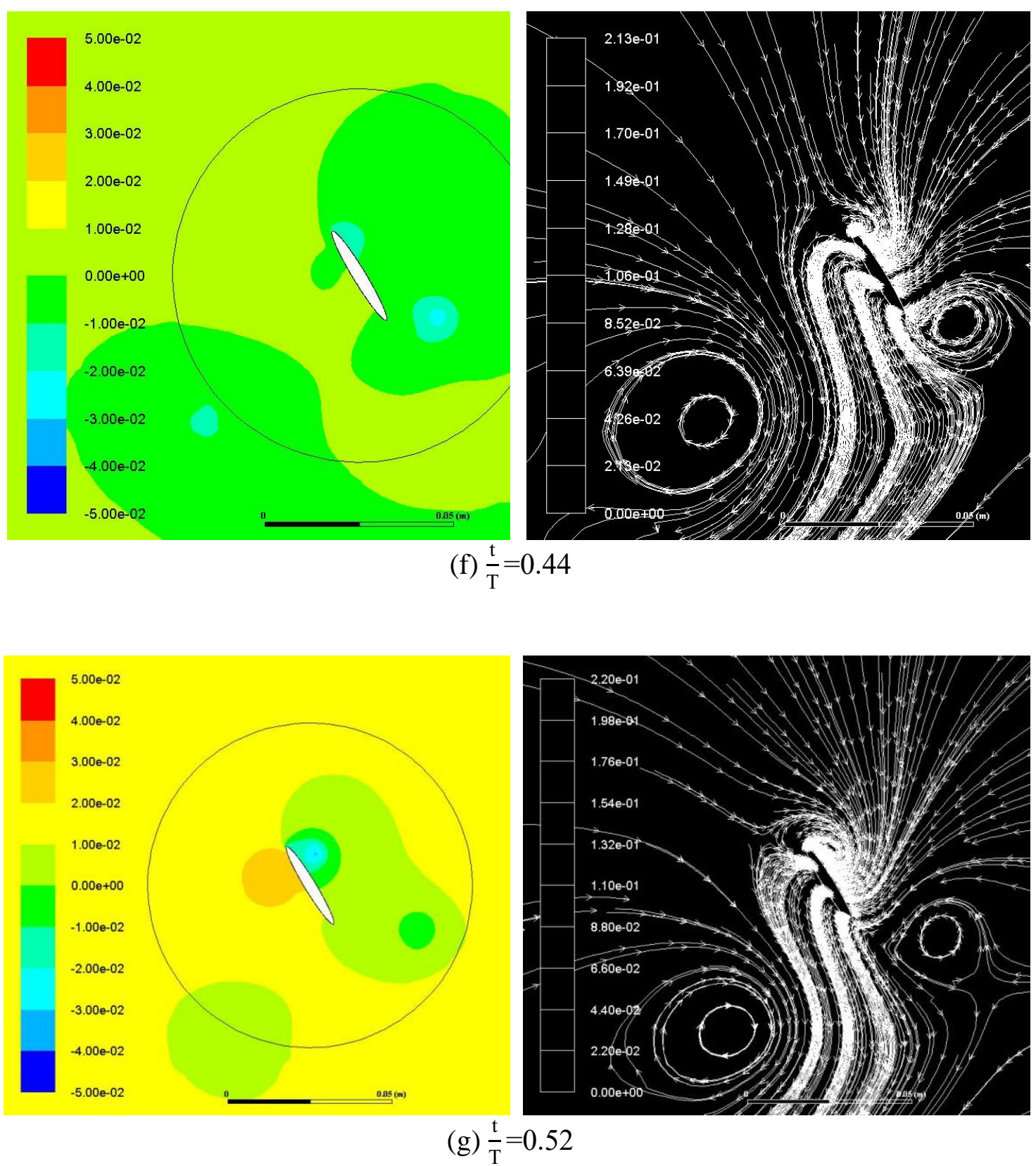

Figure 7. Flow visualisation of flow around the elliptical airfoil. The left column is the contour of static pressure, and the right column is flow pathlines.

At 0.52 the TEV leaves the airfoil while the new LEV continues to grow on the upper surface. This LEV creates a low-pressure region on the corresponding surface and hence the high value of lift coefficient (a local maximum of lift) as shown in Figure 7(g). At 0.52 , counter-rotating vortices (=vortex dipoles) are even more visible with the LEV rotates in a clockwise direction, while the TEV rotates in the opposite direction (counterclockwise).

The pressure contour and flow pathlines at the end of a cycle $\left(\frac{t}{T}=0.92\right)$ are shown in Figure 8. At this time, counter-rotating vortices have developed on the upper surface of the airfoil. These vortex dipoles draw velocity onto the upper surface. Thus a lowpressure region covers the surface as seen in Figure 8(a). The vortex dipoles and 
subsequently the low-pressure region on the upper surface cause the elliptical airfoil to generate a high value of lift $\left(\mathrm{C}_{1}=0.6996\right)$.

The relation between vortex formation and lift generation has been established by explaining the flow structures at different time instances throughout the flapping cycle/wingbeat. This finding reinforces the fact that bumblebees use the formation and shedding of vortices to produce lift enough to stay aloft while carrying a heavy load. The counter-rotating vortices captured clearly in this simulation and the established relation between vortices and lifted become an indicator of how 2D elliptical airfoils can be used as an alternative to studying 3D bumblebee wings. Simulations of 2D airfoils exclude mechanisms such as spanwise flow and tip vortices, but still, show useful information on the flow around flapping airfoils.

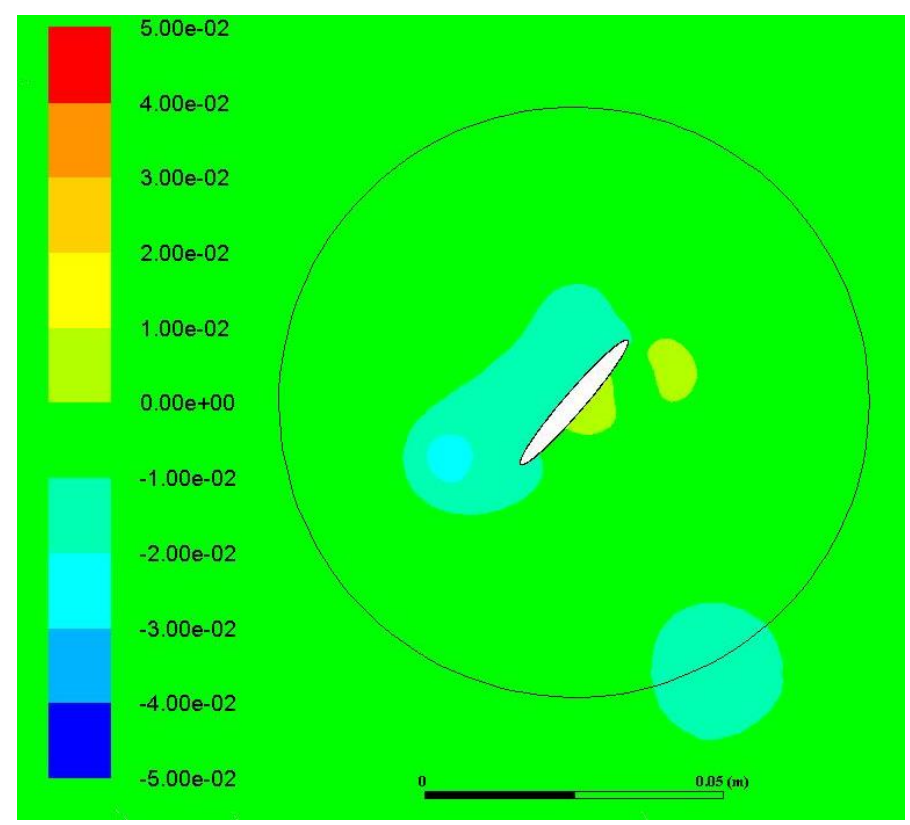

(a) contour of static pressure

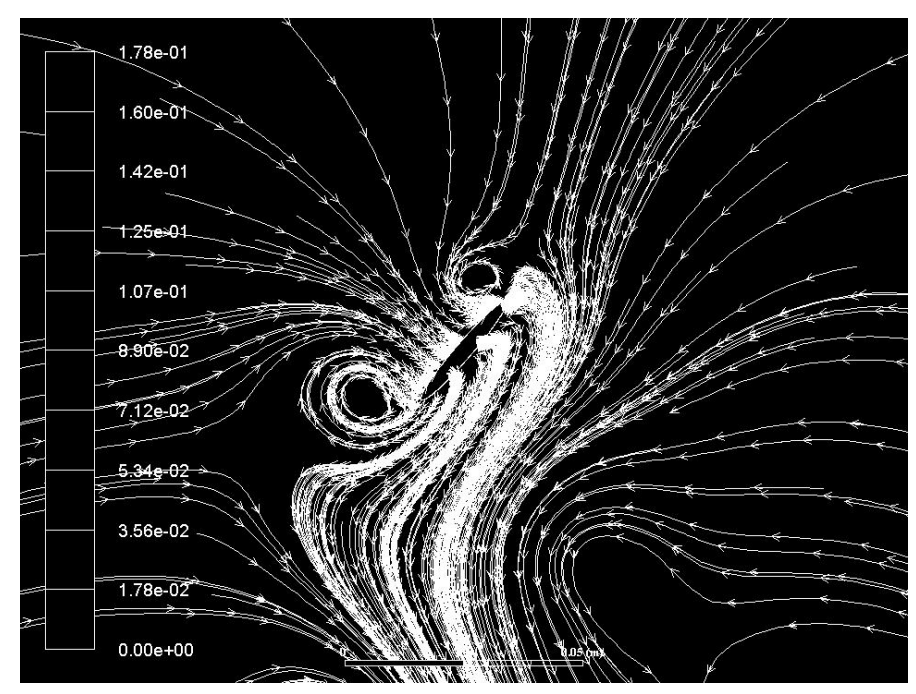

(b) flow pathlines

Figure 8. Vortex dipoles at $\frac{\mathrm{t}}{\mathrm{T}}=0.92$. 


\section{CONCLUSION}

A numerical study of flow around an elliptical airfoil that flaps under bumblebee-inspired kinematics at a Reynolds number of 1000 has been conducted. Elliptical airfoils have been analysed before but not using bumblebee flapping kinematics and typical flying Reynolds number of $\mathcal{O}\left(10^{3}\right)$. It is found that the time-averaged lift of the twodimensional elliptical airfoil is used to approximate the magnitude of lift force produced by a four-winged bumblebee.

There are three conclusions drawn from this study. Firstly, it is found that the wings of bumblebee generate enough lift to counter its weight. The excess lift indicates that the bumblebee is able to carry not only its body but also other loads such as nectar and pollen, which is something that can be observed in nature. Secondly, the counterrotating vortices are successfully captured, and a correlation between this flow structure and lift production can be established. The counter-rotating vortices are generated throughout a flapping cycle to enable the insect to produce a high magnitude of lift which allows the bumblebee to stay aloft during a hovering flight. The relation between counterrotating vortices and drag production of an elliptical airfoil at the same Reynolds number of 1000 had been preliminarily studied [26] and needs to be analysed further in future works. Thirdly, the 2D elliptical airfoil can be used to provide insight on the vortex rings on 3D bumblebee wings. Future works on bumblebee aerodynamics and propulsion can be conducted using 2D elliptical airfoil.

\section{REFERENCES}

[1] Sane SP, Dickinson MH. The aerodynamic effects of wing rotation and a revised quasi-steady model of flapping flight. Journal of Experimental Biology. 2002; 205(8): 1087.

[2] Birch JM, Dickinson MH. Spanwise flow and the attachment of the leading-edge vortex on insect wings. Nature. 2001; 412:729.

[3] Dickinson MH, Lehmann F-O, Sane SP. Wing rotation and the aerodynamic basis of insect flight. Science. 1999; 284(5422): 1954.

[4] Birch JM, Dickinson MH. The influence of wing-wake interactions on the production of aerodynamic forces in flapping flight. Journal of Experimental Biology. 2003; 206(13):2257.

[5] Chimakurthi SK, Tang J, Palacios R, Cesnik CE, Shyy W. Computational aeroelasticity framework for analyzing flapping wing micro air vehicles. American Institute of Aeronautics and Astronautics Journal. 2009; 47(8): 1865-

[6] Goli S, Roy A, Patel DK, Roy S. Particle image velocimetry measurements of rigid and flexible rectangular wings undergoing main flapping motion in hovering flight. In: Saha AK, Das D, Srivastava R, Panigrahi PK, Muralidhar K, editors. Fluid Mechanics and Fluid Power-Contemporary Research. Springer India; 2017. p 1411-20.

[7] Chandar D, Damodaran M. Computation of Low Reynolds number aerodynamic characteristics of a flapping wing in free flight. In: Choi H, Choi HG, Yoo JY, editors. Computational Fluid Dynamics 2008, Berlin: Springer, 2009. p 197-202.

[8] Truong TQ, Phan VH, Park HC, Ko JH. Effect of wing twisting on aerodynamic performance of flapping wing system. AIAA Journal. 2013; 51(7): 1612-20.

[9] Wu P, Ifju P. Micro air vehicle flapping wing effectiveness, efficiency and aeroelasticity relationships. In: 48th AIAA Aerospace Sciences Meeting Including 
the New Horizons Forum and Aerospace Exposition, Orlando, Florida; 4-7 January, 2010.

[10] Aono H, Chimakurthi S, Wu P, Sällström E, Stanford B, Cesnik C, et al. A computational and experimental study of flexible flapping wing aerodynamics. In: 48th AIAA Aerospace Sciences Meeting Including the New Horizons Forum and Aerospace Exposition, Orlando, Florida; 4-7 January, 2010.

[11] Wang ZJ. Vortex shedding and frequency selection in flapping flight. Journal of Fluid Mechanics. 2000; 410:323-41.

[12] Ashraf M, Young J, Lai J. Effect of airfoil thickness, camber and reynolds number on plunging airfoil propulsion. In: 47th AIAA Aerospace Sciences Meeting including The New Horizons Forum and Aerospace Exposition. Orlando, Florida; 5-8 January, 2009

[13] Miao J-M, Ho M-H. Effect of flexure on aerodynamic propulsive efficiency of flapping flexible airfoil. Journal of Fluids and Structures. 2006; 22(3): 401-19.

[14] Kurinami T, Fuchiwaki M, Tanaka K. Vortex structure around heaving elastic airfoils and characteristics of dynamic thrust. In: 48th AIAA Aerospace Sciences Meeting Including the New Horizons Forum and Aerospace Exposition, Orlando, Florida; 4-7 January, 2010.

[15] Yang T, Wei M, Zhao H. Numerical study of flexible flapping wing propulsion. American Institute of Aeronautics and Astronautics Journal. 2010; 48(12): 29092915.

[16] Heathcote S, Gursul I. Flexible flapping airfoil propulsion at low reynolds numbers. American Institute of Aeronautics and Astronautics Journal. 2007; 45(5): 1066-1079.

[17] Vanella M, Fitzgerald T, Preidikman S, Balaras E, Balachandran B. Influence of flexibility on the aerodynamic performance of a hovering wing. Journal of Experimental Biology. 2009; 212(1): 95.

[18] Trizila P, Kang C-K, Aono H, Shyy W, Visbal M. Low-Reynolds-number aerodynamics of a flapping rigid flat plate. AIAA Journal. 2011;49(4):806-23.

[19] Byrne DN, Buchmann SL, Spangler HG. Relationship between wing loading, wingbeat frequency and body mass in homopterous insects. Journal of Experimental Biology. 1988; 135(1):9.

[20] Bomphrey RJ, Taylor GK, Thomas ALR. Smoke visualization of free-flying bumblebees indicates independent leading-edge vortices on each wing pair. Experiments in Fluids. 2009; 46(5):811-21.

[21] Jane Wang Z. Two dimensional mechanism for insect hovering. Physical Review Letters. 2000; 85(10):2216-9. 10.1103/PhysRevLett.85.2216.

[22] Poelma C, Dickson WB, Dickinson MH. Time-resolved reconstruction of the full velocity field around a dynamically-scaled flapping wing. Experiments in Fluids. 2006; 41(2):213-25.

[23] Sun W, Gao Z, Du Y, Xu F. Mechanism of unconventional aerodynamic characteristics of an elliptic airfoil. Chinese Journal of Aeronautics. 2015; 28(3): 687-94.

[24] Wang ZJ. The role of drag in insect hovering. Journal of Experimental Biology. 2004; 207(23):4147.

[25] Mittal S, Kumar V, Raghuvanshi A. Unsteady incompressible flows past two cylinders in tandem and staggered arrangements. International Journal for Numerical Methods in Fluids. 1997; 25(11): 1315-44. 
[26] Tobing S. Drag of an elliptical airfoil at a low Reynolds number. In: International Conference on Aerospace and Aviation, Bandung, Indonesia; 23-25 October, 2018.

[27] Dudley R, Ellington CP. Mechanics of forward flight in bumblebees: I. kinematics and morphology. Journal of Experimental Biology. 1990; 148(1): 19.

[28] Tobing S, Young J, Lai JCS. Effects of wing flexibility on bumblebee propulsion. Journal of Fluids and Structures. 2017; 68: 141-57.

[29] Tobing S, Young J, Lai J. A Numerical Analysis of Bumblebee Propulsion. In: 31st AIAA Applied Aerodynamics Conference, San Diego, USA; 24-27 June, 2013. 